\title{
Evidencia de reclutamiento coralino producto de reproducción sexual, en Isla Gorgona, Pacífico Tropical Oriental
}

\section{Evidence of sexually-produced coral recruitment at Gorgona Island, Eastern Tropical Pacific}

\author{
Carlos G. Muñoz ${ }^{1,2}$, Juliana Jaramillo-González ${ }^{1}$ y Fernando A. Zapata ${ }^{1}$ \\ (iD) $0000-0001-5439-8542 \quad$ (D) $0000-0002-5720-8772 \quad$ (D) $0000-0001-8989-4458$

\begin{abstract}
${ }^{I}$ Grupo de Ecología de Arrecifes Coralinos, Universidad del Valle.Calle 13 \# 100-00.Cali, Colombia.carlos.g.munoz@correounivalle.edu.co
${ }^{2}$ Instituto de Investigaciones Marinas y Costeras - Invemar [Marine and Coastal Research Institute]. Calle 25 No. 2-55, Playa Salguero. Rodadero, Santa Marta, Colombia.
\end{abstract}

\section{RESUMEN}

$\mathrm{S}$ e presenta evidencia de reclutamiento coralino de origen sexual, el cual ha sido típicamente bajo en la región del Pacífico Tropical Oriental, en diferentes arrecifes coralinos y sitios rocosos de isla Gorgona, Colombia, y por primera vez para el Pacífico colombiano. Mediante un examen minucioso de sustratos naturales, encontramos reclutas de coral de al menos diez especies, en diez de 19 sitios alrededor de la isla durante exploraciones de buceo entre los años 2010 y 2017, pero no se encontraron reclutas adheridos a placas de asentamiento de cinco materiales diferentes instaladas en uno de los arrecifes. Las dos especies de coral con juveniles más abundantes fueron Porites panamensis y Pocillopora sp., con densidades promedio de $5.7 \pm 4.7$ y $0.07 \pm 0.11$ colonias $\mathrm{m}^{-2}( \pm \mathrm{DE})$, respectivamente; y con tamaños promedio de $2.3 \pm 0.9 \mathrm{~cm}$ y $3.5 \pm 1.0 \mathrm{~cm}( \pm \mathrm{DE})$, respectivamente. Estos resultados indican que, aunque las placas de asentamiento no son tan útiles para estudiar el reclutamiento de coral en esta región como sí lo han sido en otros lugares del mundo, el reclutamiento coralino derivado de la reproducción sexual de un conjunto diverso de especies sí es un proceso activo en la isla.

PALABRAS CLAVE: Asentamiento coralino, Reclutamiento sexual, Corales juveniles, Pocillopora, Porites.

\section{ABSTRACT}

$\mathrm{W}$ e present evidence of coral sexual recruitment, which has typically been low in the Eastern Tropical Pacific, on coral reefs and rocky sites of Gorgona Island, Colombia, and for the first time in the Colombian Pacific. With data obtained by thoroughly examining natural substrates, coral juveniles of at least ten species were found at ten out of 19 sites surveyed during scuba diving explorations performed around the island between 2010-2017; however, no corals were found on settlement plates of five different materials. The two most abundant coral species found as juveniles were Porites panamensis and Pocillopora sp., occurring at mean densities of $5.7 \pm 4.7$ and $0.07 \pm 0.11$ colonies $\mathrm{m}^{-2}( \pm \mathrm{SD})$, respectively, and with mean colony-sizes of $2.3 \pm 0.9 \mathrm{~cm}$ and $3.5 \pm 1.0 \mathrm{~cm}( \pm \mathrm{SD})$, respectively. These results indicate that, even though settlement plates do not seem to be as useful to study coral recruitment in the region as they are elsewhere, coral recruitment derived from the sexual reproduction of a diverse set of species is an active process at Gorgona Island.

KEY WORDS: Coral settlement, Sexual recruitment, Juvenile corals, Pocillopora, Porites.

DOI: 10.25268/bimc.invemar.2018.47.2.749 


\section{INTRODUCCIÓN}

El reclutamiento se define como la incorporación de nuevos individuos a una población y es un proceso demográfico esencial vinculado al mantenimiento de las poblaciones silvestres (Caley et al., 1996). En el caso de organismos como los árboles y corales, que son los respectivos componentes estructurales fundamentales de bosques y arrecifes coralinos, el reclutamiento también determina la resiliencia de estos ecosistemas (Hughes y Tanner, 2000). La comprensión del reclutamiento de corales es primordial dado el evidente deterioro global de las últimas décadas en los arrecifes de coral y sus servicios asociados (Richmond, 1997; Babcock et al., 2003; Hughes et al., 2010).

El reclutamiento de corales se ha documentado relativamente bien en las regiones del Indo-Pacífico, Pacífico Central y Caribe, y ha sido menos estudiado en el Pacífico Tropical Oriental - PTO (Glynn et al., 2017a). Los primeros estudios en esta región no encontraron reclutas de corales sobre sustratos naturales en Panamá, y muestreos realizados con placas de asentamiento resultaron en un reclutamiento extremadamente bajo o nulo (Birkeland, 1977; Wellington, 1982; Richmond, 1985, 1987). Estos resultados negativos, además de aquellos de los primeros análisis histológicos, llevaron incluso a algunos investigadores a considerar a los corales del PTO como "un ejemplo de población estéril o expatriada" (Richmond, 1985, 1987), cuya recuperación de las perturbaciones era producto principalmente de la reproducción asexual, especialmente por fragmentación de colonias (Highsmith, 1982; Muñoz et al., 2015). Estudios más recientes han encontrado pocos -si es que han encontrado alguno- reclutas en las placas de asentamiento, por ejemplo: nueve reclutas de Porites en el Pacífico mexicano (MedinaRosas et al., 2005), 291 reclutas de Porites panamensis, pero sólo un recluta de Pocillopora también en el Pacífico mexicano (López-Pérez et al., 2007), y ningún recluta en la isla Gorgona, Pacífico colombiano (Lozano-Cortés y Zapata, 2014). Además, los nuevos análisis histológicos indicaban claramente que la maduración gonadal era un proceso activo (Glynn et al., 1991, 1994, 2017a y sus referencias), incluso en los corales pocilopóridos de isla Gorgona (Castrillón et al., 2015).

Aunque la isla Gorgona se encuentra dentro de un área marina protegida en la que prácticamente no existen impactos antropogénicos directos, estos arrecifes se ven afectados negativamente por perturbaciones naturales recurrentes como el calentamiento temporal del agua por el fenómeno de El Niño-Oscilación del Sur y las exposiciones al aire durante mareas extremadamente bajas (Zapata y Vargas-Ángel, 2003;

\section{INTRODUCTION}

Recruitment, the incorporation of new individuals to a population, is a key demographic process involved in the maintenance of wild populations (Caley et al., 1996). In the case of organisms like trees and corals, key structural components of forests and coral reefs, recruitment also determines the resilience of these ecosystems (Hughes and Tanner, 2000). Understanding coral recruitment is critical given the evident global deterioration of coral reefs and their associated services in recent decades (Richmond, 1997; Babcock et al., 2003; Hughes et al., 2010).

Coral recruitment has been relatively well documented in the Indo-Pacific, Central Pacific and Caribbean regions, and less studied in the Eastern Tropical Pacific - ETP (Glynn et al., 2017a). Early studies in the ETP did not find coral recruitment on natural substrates, and sampling with settlement plates resulted in extremely low to nil recruitment (Birkeland, 1977; Wellington, 1982; Richmond, 1985; 1987). Such negative results, in addition to those from early histological analyses, even led some researchers to consider ETP corals as "an example of a sterile or expatriate population" (Richmond, 1985, 1987), whose recovery from perturbations was primarily driven by asexual reproduction such as that resulting from colony fragmentation (Highsmith, 1982; Muñoz et al., 2015). More recent studies found few - if any recruits on settlement plates, for example: nine Porites recruits in the Mexican Pacific (Medina-Rosas et al., 2005), 291 recruits of Porites panamensis but only one Pocillopora recruit also in the Mexican Pacific (LópezPérez et al., 2007), and no recruits at all in Gorgona Island, Colombian Pacific (Lozano-Cortés and Zapata, 2014). Furthermore, new histological evidence clearly indicated that gonadal maturation was an active process (Glynn et al., 1991; 1994; 2017a and references therein), including pocilloporids at Gorgona Island (Castrillón et al., 2015).

Although Gorgona Island is located within a marine protected area so that direct anthropogenic impacts are virtually absent, these reefs are negatively affected by recurring natural disturbances such as El Niño warming events and subaerial exposures during extreme low tides (Zapata and Vargas-Ángel, 2003; Castrillón et al., 2017; 
Castrillón et al., 2017; Fiedler y Lavín, 2017). Por ejemplo, la cobertura de coral vivo en el arrecife de La Azufrada disminuyó en promedio $26 \%$ durante la década 1998-2008, siendo aún más crítico en las áreas someras del arrecife donde pasó de $61 \%$ a 15\% (Zapata, 2017), a partir de donde ha ido recuperándose lentamente. Para entender apropiadamente estos cambios en el ecosistema, es urgente conocer en qué medida contribuye el reclutamiento de los corales a la resiliencia de estos arrecifes; sin embargo, una dificultad importante para lograrlo ha sido la falta de evidencia de que el reclutamiento coralino producto de la reproducción sexual ocurre en la isla.

En este estudio, se presenta evidencia del reclutamiento de corales producto del asentamiento de larvas en sustratos naturales de arrecifes coralinos y sitios rocosos de la isla Gorgona, registrando al mismo tiempo la ausencia de reclutas de coral en placas de asentamiento de diferentes materiales. También se registra la distribución de frecuencia de tamaños y la densidad poblacional de colonias juveniles de Pocillopora sp. y Porites panamensis.

\section{MÉTODOS}

\section{Área de estudio}

Isla Gorgona se encuentra ubicada a unos 30 $\mathrm{km}$ de la costa $\left(3^{\circ} 00^{\prime} 55^{\prime \prime} \mathrm{N}, 78^{\circ} 14^{\prime} 30^{\prime \prime} \mathrm{O}\right)$ y constituye el territorio insular más grande $\left(13.2 \mathrm{~km}^{2}\right)$ de la plataforma continental del Pacífico colombiano. La isla y sus aguas circundantes son parte del Parque Nacional Natural Gorgona, que alberga algunas de las formaciones de arrecifes de coral más grandes, diversas y desarrolladas del PTO, junto con extensas costas rocosas intermareales y montañas rocosas sumergidas (Zapata y Vargas-Ángel, 2003; Muñoz y Zapata, 2013). Hasta la fecha, se conocen alrededor de 25 especies de corales hermatípicos en la isla, pero debido a las dificultades taxonómicas dentro del género Pocillopora (Schmidt-Roach et al., 2014), el número verdadero aún se desconoce (Glynn et al., 2017b). Los corales pocilopóridos dominan en las aguas poco profundas ( $<5 \mathrm{~m}$ de profundidad) y son los principales constructores de los verdaderos arrecifes coralinos, mientras que las especies de crecimiento masivo como Pavona varians, Porites lobata y Gardineroseris planulata están relegadas a los bordes exteriores de esos arrecifes pocilopóridos, siendo más comunes en sitios rocosos más profundos. Las especies de coral más frecuentes en isla Gorgona se consideran liberadoras de gametos, excepto por Porites panamensis que es incubadora, y por las especies de Pocillopora, las cuales han mostrado diversas formas de reproducción en todo el mundo (Glynn et al., 1991).
Fiedler and Lavín, 2017). For example, mean live coral cover at La Azufrada reef declined 26\% in the decade 1998 2008 , and critically so in shallow areas where it decreased from $61 \%$ to $15 \%$ (Zapata, 2017). Therefore, it has become urgent to know how resilient these reefs are and how much coral recruitment contributes to their resilience. However, one major difficulty to accomplish this has been the lack of evidence showing that sexually-produced coral recruitment actually occurs at Gorgona Island.

In this study, we present evidence of coral recruitment derived from larval settlement on natural substrates in coral reefs and rocky sites at Gorgona Island, but report the absence of coral recruits on settlement plates of different materials. We also report the population densities and size frequency distributions of juvenile colonies of Pocillopora sp. and Porites panamensis.

\section{METHODS}

\section{Study area}

Gorgona Island, located $\sim 30 \mathrm{~km}$ off the coast $\left(3^{\circ} 00^{\prime}\right.$ $\left.55^{\prime \prime} \mathrm{N}, 78^{\circ} 14^{\prime} 30^{\prime \prime} \mathrm{W}\right)$, constitutes the largest insular territory $\left(13.2 \mathrm{~km}^{2}\right)$ in the Colombian Pacific continental shelf. The Island and its surrounding waters are part of Gorgona National Natural Park, which harbors some of the largest, most diverse and developed coral reef formations in the ETP, along with extensive intertidal rocky shorelines and submerged rocky mounts (Zapata and Vargas-Ángel, 2003; Muñoz and Zapata, 2013). To date, around 25 species of hermatypic coral are known from Gorgona Island, but due to taxonomic difficulties within the genus Pocillopora (Schmidt-Roach et al., 2014), the true number remains uncertain (Glynn et al., 2017b). Pocilloporid corals dominate the shallow waters ( $<5 \mathrm{~m} \mathrm{depth})$ and are the main builders of the coral reefs, while massive-growth species like Pavona varians, Porites lobata and Gardineroseris planulata are common in the forereef zones and in deeper rocky sites. Most coral species in Gorgona Island are considered broadcast spawners, except Porites panamensis that is a brooder, and the pocilloporid species with diverse reproductive strategies around the world (Glynn et al., 1991).

The average annual rainfall is high ranging between $4000-7000 \mathrm{~mm}$ with two contrasting climatological seasons, a rainy one from May to October and a dry season between December and March (Diaz 
La precipitación anual promedio es alta, oscilando entre 4000 - $7000 \mathrm{~mm}$ con dos estaciones climatológicas contrastantes, una lluviosa de mayo a octubre y otra seca de diciembre a marzo (Díaz et al., 2001; Blanco, 2009). Debido a las fuertes lluvias en Gorgona, las cuerpos de agua dulce son abundantes y la turbidez del mar es muy variable y está parcialmente relacionada con la lluvia. El régimen de mareas es semidiurno, con un ámbito máximo de marea de 5.7 m (IDEAM, 2017); las corrientes oceánicas y la acción del oleaje son más fuertes en el lado oeste, barlovento de la isla principal (Giraldo et al., 2008).

\section{Muestreo}

Se implementaron tres métodos diferentes para estudiar el reclutamiento coralino producto de la reproducción sexual en Isla Gorgona: A) una amplia búsqueda de corales juveniles en los sustratos naturales alrededor de la isla durante inmersiones exploratorias, B) conteos visuales de los corales juveniles en transectos, y C) el uso de placas de asentamiento para muestrear reclutas de coral.

\section{Inmersiones exploratorias}

Durante las inmersiones exploratorias en busca de corales juveniles, se inspeccionaron un total de 19 sitios alrededor de la isla, incluyendo arrecifes de coral y sitios rocosos. Se realizaron al menos tres inmersiones en cada sitio al año; se examinaron 17 sitios durante 2010 - 2015, y dos sitios adicionales durante 2017.

Se utilizaron diferentes criterios (basados en Richmond, 1985 y Glynn et al., 1996) para determinar si las pequeñas colonias de coral eran el resultado del asentamiento de larvas, particularmente en los pocilopóridos ramificados que se fragmentan con frecuencia (Highsmith, 1982; Glynn et al., 1991; Muñoz et al., 2015). Los criterios fueron: 1) La presencia de una base incrustante ancha, plana y casi simétrica, 2) la fijación a sustratos empinados o verticales, como las paredes rocosas, donde no se esperaría encontrar fragmentos de coral, y 3) la ubicación de los corales juveniles suficientemente alejados de las colonias adultas. El cumplimiento simultáneo de algunos criterios para la identificación de corales juveniles ha supuesto un fuerte apoyo frente a las críticas de algunos autores de que la inspección visual de los corales juveniles no es suficiente para establecer el origen sexual (por asentamiento de una larva) o asexual (por supervivencia de un fragmento) de una pequeña colonia de coral (ej., Miller et al., 2008).

Similar a Edmunds (2004), un criterio usado para definir a los corales juveniles fue un tamaño de colonia entre et al., 2001; Blanco, 2009). Due to the heavy rainfall at Gorgona, freshwater streams are abundant, and turbidity is highly variable and partially related to rainfall. Tidal regime is semidiurnal, with a maximum tidal range of 5.7 m (IDEAM, 2017); ocean currents and wave action are stronger on the western, windward side of the main island (Giraldo et al., 2008).

Sampling

Three different methods to examine recruitment of sexually-produced corals at Gorgona Island were implemented: A) a wide search for coral juveniles on natural substrates around the island during exploratory dives, B) visual counts of coral juveniles on transects, and C) the use of settlement plates to sample coral recruits.

\section{Exploratory dives}

A total of 19 sites around the island including both coral reefs and rocky sites were inspected during exploratory dives searching for juvenile corals. Minimum three dives at each site were performed per year, 17 sites were surveyed during 2010 - 2015, and two additional sites were surveyed during 2017.

Different criteria (based on Richmond, 1985 and Glynn et al., 1996) were used to establish whether small coral colonies were derived from larval settlement, particularly in the branching pocilloporids that fragment frequently (Highsmith, 1982; Glynn et al., 1991; Muñoz et al., 2015). 1) The presence of a wide, flat, and nearlysymmetrical encrusting base, 2) attachment to steep or vertical substrates like rocky walls, where coral fragments would not be expected to occur, and 3) location of juveniles sufficiently far from adult colonies. Simultaneously fulfilling our criteria for each juvenile provided strong support against criticism by some authors that visual inspection of coral juveniles is insufficient to establish the sexual or asexual origin of a small coral colony (e.g., Miller et al., 2008).

Similar to Edmunds (2004), a criterion to define coral juveniles was a colony-size between $0.1 \mathrm{~cm}(1$ polyp) and $\leq 5.0 \mathrm{~cm}$ diameter, hence including both recruits and juvenile corals. Considering that pocilloporid corals achieve sexual maturity at around 2-3 years old and grow $\sim 2.7 \mathrm{~cm} \mathrm{y}^{-1}$ (Palacios et al., 2014), a colony $5 \mathrm{~cm}$ in diameter would be 1.5 years old. Similarly, massive corals like Porites panamensis would be approximately 3.5 years old considering that they reach maturity at 4-7 years 
$\sim 0.1 \mathrm{~cm}$ (1 pólipo) y $\leq 5.0 \mathrm{~cm}$ de diámetro, incluyendo por lo tanto reclutas y corales juveniles. Considerando que los corales pocilopóridos alcanzan la madurez sexual alrededor de los 2-3 años y crecen $\sim 2.7 \mathrm{~cm} \mathrm{a}^{-1}$ (Palacios et al., 2014), una colonia de $5 \mathrm{~cm}$ de diámetro tendría 1.5 años de edad. Del mismo modo, los corales masivos como Porites panamensis tendrían aproximadamente 3.5 años de edad, considerando que alcanzan la madurez a los 4-7 años (Richmond, 1997) y crecen $\sim 1.5 \mathrm{~cm} \mathrm{a}^{-1}$ (Cabral-Tena et al., 2013). Finalmente, debido a que los corales juveniles pocilopóridos fueron difíciles de identificar a nivel de especie, se clasificaron como Pocillopora sp.

\section{Conteo en transectos}

Para estimar la abundancia y el tamaño (diámetro máximo) de las especies de corales juveniles, durante noviembre de 2010 se examinaron diez transectos visuales (cada transecto de $20 \mathrm{~m}^{2}$ ) en cada uno de los dos arrecifes de coral más grandes de la isla (La Azufrada y Playa Blanca); además, durante marzo de 2011 se examinaron nueve transectos similares en la costa rocosa de El Laberinto; y durante junio de 2011, se examinaron tres transectos más pequeños $\left(10 \mathrm{~m}^{2}\right)$ en el arrecife coralino El Arrecifito. Para evaluar la abundancia y las diferencias de tamaño entre los corales juveniles de pocilopóridos de diferentes sitios, se utilizó ANOVA de una vía, después de comprobar que se cumplían los supuestos de homocedasticidad y normalidad.

\section{Placas de asentamiento}

En octubre de 2010, se instalaron en total 54 placas de asentamiento de $\sim 20 \times 20 \times 0.5 \mathrm{~cm}\left(\sim 840 \mathrm{~cm}^{2}\right.$ de superficie total por placa) en la planicie arrecifal $(\sim 0.5$ a $1 \mathrm{~m} \mathrm{de}$ profundidad en relación con el promedio del nivel del agua en marea baja) del arrecife de La Azufrada (12 placas de cada uno de los cuatro materiales: acrílico, cerámica, concreto y mármol, y seis placas adicionales de hilo de nylon trenzado y entretejido sirviendo de superficie en un marco de tubos de polivinilo). Las placas de los cuatro materiales acrílico, cerámica, concreto y mármol se instalaron en pares, una encima de la otra separadas aproximadamente por $2 \mathrm{~cm}$, para proporcionar entre el par de placas un microhábitat críptico a menudo preferido para el asentamiento de las larvas de coral (Harriott y Fisk, 1987); todos los pares de placas se mantuvieron unidos por una barra de acero inoxidable clavada en el sustrato. Para reducir los efectos potenciales sobre los reclutas de coral debido a la competencia por el espacio con algas y cirrípedos (Birkeland, 1977), estos organismos se eliminaron raspando las superficies old (Richmond, 1997) and grow $\sim 1.5 \mathrm{~cm} \mathrm{y}^{-1}$ (CabralTena et al., 2013). Finally, pocilloporid juveniles were difficult to identify to species, therefore, were classified as Pocillopora sp.

\section{Counts on transects}

To estimate the abundance and size (maximum diameter) of species of coral juveniles, during November 2010 ten visual transects (each transect $20 \mathrm{~m}^{2}$ ) were examined on each of two coral reefs (La Azufrada and Playa Blanca); during March 2011 nine similar transects were examined at El Laberinto rocky shore; and during June 2011 three smaller transects $\left(10 \mathrm{~m}^{2}\right)$ were examined at El Arrecifito coral reef. To test for abundance and size differences between pocilloporid juveniles from different sites, we used a one-way ANOVA, after testing for compliance with homoscedasticity and normality assumptions.

\section{Settlement plates}

In October 2010, 54 settlement plates measuring $\sim 20 \times 20 \times 0.5 \mathrm{~cm}\left(\sim 840 \mathrm{~cm}^{2}\right.$ total surface per plate $)$ were installed on the reef flat $(\sim 0.5$ to $1 \mathrm{~m}$ depth relative to the Mean Lower Low Water datum) of La Azufrada reef (twelve plates of each of four materials: acrylic, ceramic, concrete and marble; and six additional plates of tarred twisted nylon twine interwoven on a polyvinyl pipe frame). The acrylic, ceramic, concrete, and marble plates were arranged in pairs, one on top of the other separated by approximately $2 \mathrm{~cm}$ (to provide the cryptic microhabitat often preferred by settling coral larvae (Harriott and Fisk, 1987) and held together by a steel bar driven into the substrate. To reduce potential effects on coral recruits by competition for space with algae and barnacles (Birkeland, 1977), these organisms were removed by scraping the surfaces approximately every 45 days from half of the plates, which were randomly chosen the first time. All plates were photographed, with a high-resolution digital camera, approximately every 45 days for a period of six months and the photographs were examined visually on a computer. In April 2011, the plates were recovered from the sea and examined in the laboratory with a dissecting microscope.

\section{RESULTS}

\section{Exploratory dives}

We found sexually produced coral juveniles at ten out of a total of 19 sites surveyed at Gorgona Island between 2010 and 2017. Coral juveniles were observed 
de la mitad de las placas (27 placas elegidas al azar y marcadas) después de revisarlas y fotografiarlas, durante visitas al sitio de estudio aproximadamente cada 45 días. Todas las placas fueron examinadas directamente y fotografiadas con una cámara digital de alta resolución con la misma frecuencia por un período de seis meses; las fotografías fueron finalmente examinadas visualmente en un computador. En abril de 2011, se extrajeron las placas del mar y se examinaron directamente en el laboratorio con un microscopio de disección.

\section{RESULTADOS}

\section{Inmersiones exploratorias}

Encontramos corales juveniles producto de la reproducción sexual en diez de un total de 19 sitios inspeccionados en isla Gorgona entre 2010 y 2017. Se observaron corales juveniles en todos los arrecifes de coral y en algunos de los sitios rocosos. Los Farallones y El Arrecifito fueron los sitios con más especies (seis y cinco especies respectivamente, Figura 1), mientras que en los principales arrecifes de coral (La Azufrada, Playa Blanca, La Ventana, El Muelle) sólo se encontraron dos especies. Los corales juveniles pertenecían a por lo menos diez especies diferentes y cuatro familias (Tabla 1, Figura 2).

\section{Conteo en transectos}

Se observaron corales juveniles de al menos dos especies asentados sobre sustratos naturales en los transectos realizados en isla Gorgona. La especie Pocillopora sp. se encontró en los arrecifes de coral de La Azufrada y Playa Blanca, y en la costa rocosa de El Laberinto (Figuras 2A, 2B y $2 \mathrm{C}$; Tabla 1), mientras que Porites panamensis se encontró en el arrecife coralino El Arrecifito (Figura 2D, Tabla 1).

Encontramos un total de 41 ejemplares de corales juveniles pocilopóridos, estimándose una densidad media de $0.07 \pm 0.11$ colonias $\mathrm{m}^{-2}( \pm \mathrm{DE})$. El mayor reclutamiento de corales se observó en La Azufrada $(0.09 \pm 0.12$ colonias $\left.\mathrm{m}^{-2}\right)$, seguido de El Laberinto $\left(0.08 \pm 0.11\right.$ colonias $\left.\mathrm{m}^{-2}\right) \mathrm{y}$ Playa Blanca $\left(0.05 \pm 0.08\right.$ colonias $\left.\mathrm{m}^{-2}\right)$; y no se encontraron diferencias significativas en la densidad de corales juveniles de Pocillopora sp. entre los tres sitios $\left(\mathrm{F}_{2,26}=0.28, \mathrm{p}>0.1\right)$. El tamaño medio de colonia de estos corales juveniles fue de $3.5 \pm 1.0 \mathrm{~cm}$, sin diferencias significativas entre los tres sitios $\left(\mathrm{F}_{2,38}=0.31 ; \mathrm{p}>0.1\right.$; Figura 3A, 3B, 3C). El juvenil pocilopórido de menor tamaño medía $\sim 1.0 \mathrm{~cm}$ de diámetro y se observó en El Laberinto, asentado sobre una superficie rocosa vertical (similar a la Figura 2C). in all the coral reefs and in some of the rocky sites. Los Farallones and El Arrecifito were the sites with most species (6 and 5 species, respectively; Figure 1), while the main coral reefs (La Azufrada, Playa Blanca, La Ventana, El Muelle) had only two species. Coral juveniles belonged to at least ten different species and four families (Table 1; Figure 2).

\section{Transect counts}

Coral juveniles of at least two species were observed attached to the natural substrates within transects in Gorgona Island. Pocillopora sp. was found on the coral reefs of $\mathrm{La}$ Azufrada, and Playa Blanca, as well as at El Laberinto rocky shore (Figures 2A, 2B, 2C; Table 1), while Porites panamensis was found at El Arrecifito coral reef (Figure 2D; Table 1).

We found in total 41 pocilloporid juveniles, and estimated a mean density of $0.07 \pm 0.11$ colonies $\mathrm{m}^{-2}$ (mean $\pm \mathrm{SD}$ ). The highest coral recruitment was observed at La Azufrada $\left(0.09 \pm 0.12\right.$ colonies $\left.\mathrm{m}^{-2}\right)$, followed by El Laberinto $\left(0.08 \pm 0.11\right.$ colonies $\left.\mathrm{m}^{-2}\right)$ and Playa Blanca $\left(0.05 \pm 0.08\right.$ colonies $\left.\mathrm{m}^{-2}\right)$, but we found no significant differences in the density of pocilloporid juveniles between the three sites $\left(\mathrm{F}_{2,26}=0.28, \mathrm{p}>0.1\right)$. The mean colonysize of pocilloporid juveniles was $3.5 \pm 1.0 \mathrm{~cm}$, also with no significant differences between the three sites $\left(\mathrm{F}_{2,38}=\right.$ $0.31 ; p>0.1$; Figure 3A, 3B, 3C). The smallest pocilloporid juvenile had a diameter $\sim 1.0 \mathrm{~cm}$ and was observed at $\mathrm{El}$ Laberinto attached to a vertical rocky surface (similar to Figure 2C).

At El Arrecifito, we found a total of 170 juveniles of Porites panamensis with a mean density of $5.7 \pm 4.7$ colonies $\mathrm{m}^{-2}$ and a mean colony size of $2.3 \pm 0.9 \mathrm{~cm}$. The smallest $P$. panamensis colony was $\sim 0.5 \mathrm{~cm}$ in diameter, and had between 15 to 20 polyps each one about $0.1 \mathrm{~cm}$ in diameter (Figure 2D; 3D).

\section{Settlement plates}

In contrast with natural substrates, we did not find coral juveniles or recruits attached to the settlement plates upon completion of the analysis of photographs or after a thorough direct examination of the plates with a dissecting microscope.

\section{DISCUSSION}

This study reports for the first time the occurrence of coral recruitment of sexual origin in the Colombian Pacific. However, we obtained two conflicting 


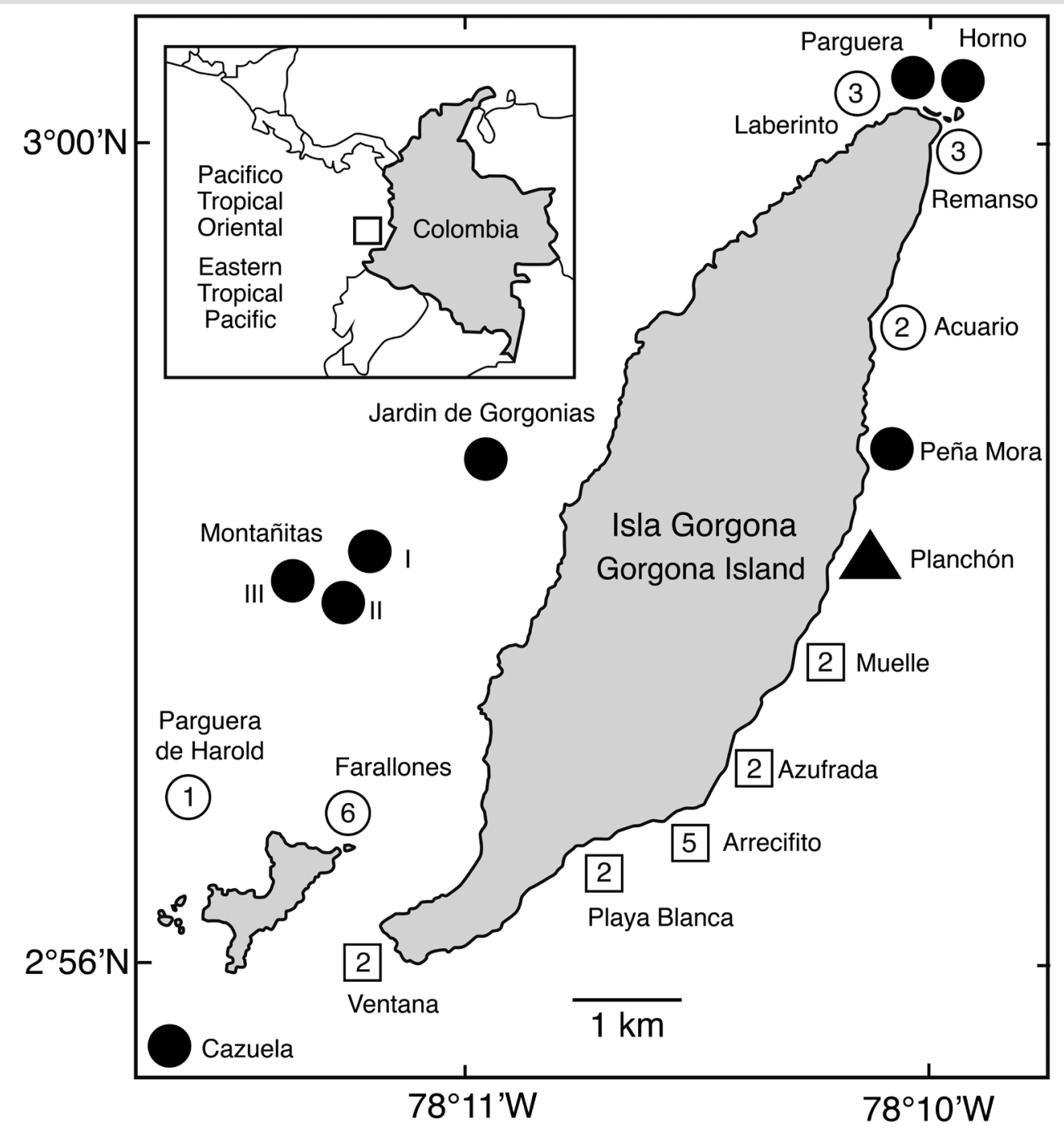

Figura 1. Número de especies de corales juveniles por sitio. Cuadrado = arrecife de coral; círculo = sitio rocoso; triángulo = naufragio en fondo arenoso. Negro $=$ no se encontraron corales juveniles.
Figure 1. Number of species of juvenile corals per site. Square $=$ coral reef; circle $=$ rocky site; triangle $=$ shipwreck on sandy bottom. Black $=$ no juvenile corals found.
Tabla 1. Presencia de los corales juveniles observados en el Parque Nacional Natural Gorgona (Colombia, Pacífico Tropical Oriental) durante 2010 - 2017.

\begin{tabular}{|c|c|c|c|c|c|c|c|c|c|c|c|c|c|}
\hline & & & & \multicolumn{6}{|c|}{ Agariciidae } & \multirow{2}{*}{ 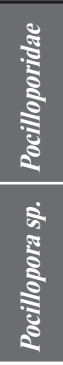 } & \multicolumn{2}{|c|}{ Poritidae } & \multirow{2}{*}{ 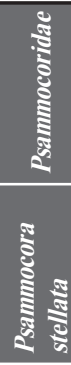 } \\
\hline $\begin{array}{l}\text { Sitio } \\
\text { Site }\end{array}$ & $\begin{array}{c}\text { Ámbito de } \\
\text { profundidad (m) } \\
\text { Depth Range (m) }\end{array}$ & $\begin{array}{c}\text { Cobertura } \\
\text { de coral } \\
(\sim \%) \\
\text { Coral cover } \\
(\sim \%)\end{array}$ & $\begin{array}{c}\text { Años del } \\
\text { estudio } \\
\text { Study years }\end{array}$ & 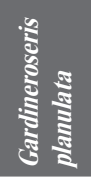 & 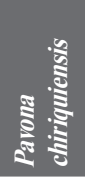 & $\frac{\sqrt{2}}{\frac{2}{3}}$ & 言 & 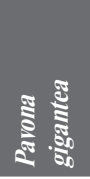 & 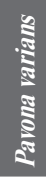 & & 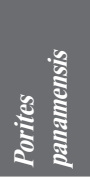 & 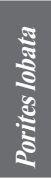 & \\
\hline \multicolumn{14}{|c|}{$\begin{array}{l}\text { Arrecifes de coral } \\
\text { Coral Reefs }\end{array}$} \\
\hline Arrecifito & $0-10$ & $70.0 \%$ & 2010-2012 & - & - & - & - & $\mathrm{X}$ & $\mathrm{X}$ & $X$ & $\mathrm{X}$ & - & $\mathrm{X}$ \\
\hline Azufrada & $0-10$ & $50.0 \%$ & $2010-2015$ & - & - & - & - & - & $\mathrm{X}$ & $X$ & - & - & - \\
\hline Muelle & $0-10$ & $70.0 \%$ & $2010-2015$ & - & - & - & - & $X$ & $\mathrm{X}$ & - & - & - & - \\
\hline
\end{tabular}




\begin{tabular}{|c|c|c|c|c|c|c|c|c|c|c|c|c|c|}
\hline & & & & \multicolumn{6}{|c|}{ Agariciidae } & \multirow{2}{*}{ 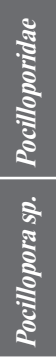 } & \multicolumn{2}{|c|}{ Poritidae } & \multirow{2}{*}{ 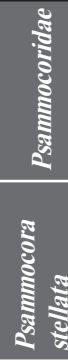 } \\
\hline $\begin{array}{l}\text { Sitio } \\
\text { Site }\end{array}$ & $\begin{array}{c}\text { Ámbito de } \\
\text { profundidad (m) } \\
\text { Depth Range (m) }\end{array}$ & $\begin{array}{c}\text { Cobertura } \\
\text { de coral } \\
(\sim \%) \\
\text { Coral cover } \\
(\sim \%)\end{array}$ & $\begin{array}{c}\text { Años del } \\
\text { estudio } \\
\text { Study years }\end{array}$ & 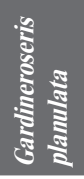 & 竎 & $\begin{array}{c}\frac{5}{5} \\
\frac{\pi}{5} \\
\frac{\pi}{5}\end{array}$ & 蛋 & 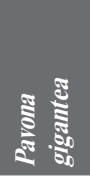 & $\frac{\sqrt{3}}{\sqrt[3]{\frac{\pi}{3}}}$ & & 总 & $\frac{\sqrt{5}}{\frac{5}{5}}$ & \\
\hline Playa Blanca & $0-10$ & $70.0 \%$ & $2010-2015$ & - & - & - & - & - & - & $\mathrm{X}$ & $X$ & - & - \\
\hline Ventana & $2-10$ & $85.0 \%$ & $2010-2015$ & $X$ & - & - & - & - & - & $\mathrm{X}$ & - & - & - \\
\hline \multicolumn{14}{|c|}{$\begin{array}{l}\text { Montañas } \\
\text { rocosas/peñascos }\end{array}$} \\
\hline \multicolumn{14}{|c|}{$\begin{array}{l}\text { Rocky mounts / } \\
\text { boulders }\end{array}$} \\
\hline Cazuela & $16-30$ & $0.5 \%$ & 2013-2014 & - & - & - & - & - & - & - & - & - & - \\
\hline Farallones & $0-12$ & $5.0 \%$ & 2010 & - & $\mathrm{X}$ & $X$ & $\mathrm{X}$ & - & - & $\mathrm{X}$ & - & $\mathrm{X}$ & $\mathrm{X}$ \\
\hline Horno & $0-35$ & $0.5 \%$ & 2013-2014 & - & - & - & - & - & - & - & - & - & - \\
\hline $\begin{array}{l}\text { Jardín de } \\
\text { Gorgonias }\end{array}$ & $10-25$ & $1.0 \%$ & 2017 & - & - & - & - & - & - & - & - & - & - \\
\hline $\begin{array}{l}\text { Parguera de } \\
\text { Harold }\end{array}$ & $10-20$ & $3.5 \%$ & 2017 & - & - & - & - & - & - & $\mathrm{X}$ & - & - & - \\
\hline Montañita I & $7-25$ & $0.5 \%$ & 2013-2014 & - & - & - & - & - & - & - & - & - & - \\
\hline Montañita II & $3-25$ & $0.5 \%$ & 2013-2014 & - & - & - & - & - & - & - & - & - & - \\
\hline Montañita III & $20-40$ & $0.5 \%$ & 2013-2014 & - & - & - & - & - & - & - & - & - & - \\
\hline Peña Mora & $7-20$ & $5.0 \%$ & 2013-2014 & - & - & - & - & - & - & - & - & - & - \\
\hline \multicolumn{14}{|l|}{ Costas rocosas } \\
\hline \multicolumn{14}{|l|}{ Rocky shores } \\
\hline Acuario & $0-5$ & $40.0 \%$ & 2010-2014 & - & - & - & - & $\mathrm{X}$ & - & $\mathrm{X}$ & - & - & - \\
\hline Laberinto & $0-15$ & $25.0 \%$ & 2010-2014 & $\mathrm{X}$ & - & - & - & - & - & $\mathrm{X}$ & - & $\mathrm{X}$ & - \\
\hline Parguera & $0-12$ & $35.0 \%$ & 2013-2014 & - & - & - & - & - & - & - & - & - & - \\
\hline Remanso & $0-12$ & $45.0 \%$ & 2010-2014 & - & - & - & - & $\mathrm{X}$ & - & $\mathrm{X}$ & - & $\mathrm{X}$ & - \\
\hline \multicolumn{14}{|l|}{ Naufragio } \\
\hline \multicolumn{14}{|l|}{ Shipwreck } \\
\hline Planchón & $5-17$ & $2.0 \%$ & 2013-2014 & - & - & - & - & - & - & - & - & - & - \\
\hline
\end{tabular}

En El Arrecifito, se encontró un total de 170 corales juveniles de Porites panamensis, con una densidad media de $5.7 \pm 4.7$ colonias $\mathrm{m}^{-2} \mathrm{y}$ un tamaño medio de colonia de $2.3 \pm 0.9 \mathrm{~cm}$. La colonia más pequeña de $P$. panamensis medía $\sim 0.5 \mathrm{~cm}$ de diámetro, y tenía entre 15 y 20 pólipos con un diámetro cada uno $\sim 0.1 \mathrm{~cm}$ (Figura 2D; 3D).

\section{Placas de asentamiento}

En contraste con los sustratos naturales, no se encontraron corales juveniles o reclutas asentados sobre las placas de asentamiento durante las visitas al sitio de estudio, ni al completar el análisis de las fotografías, ni tampoco después de un minucioso examen directo de las placas con un microscopio de disección. results similar to those reported by previous studies on coral recruitment in the ETP: despite low densities, coral juveniles were found on natural substrates on coral reefs and rocky sites, but the usual sampling method of settlement plates failed to detect coral recruitment (Birkeland, 1977; Wellington, 1982; Richmond, 1985; Medina-Rosas et al, 2005; López-Pérez et al., 2007; Lozano-Cortés and Zapata, 2014). In coral reefs, coral juveniles were found attached either to coral rubble or to the consolidated coral reef matrix covered by calcareous algae, while in rocky sites the juveniles were found attached to rocky walls, either bare or partially covered by mats of filamentous algae. 

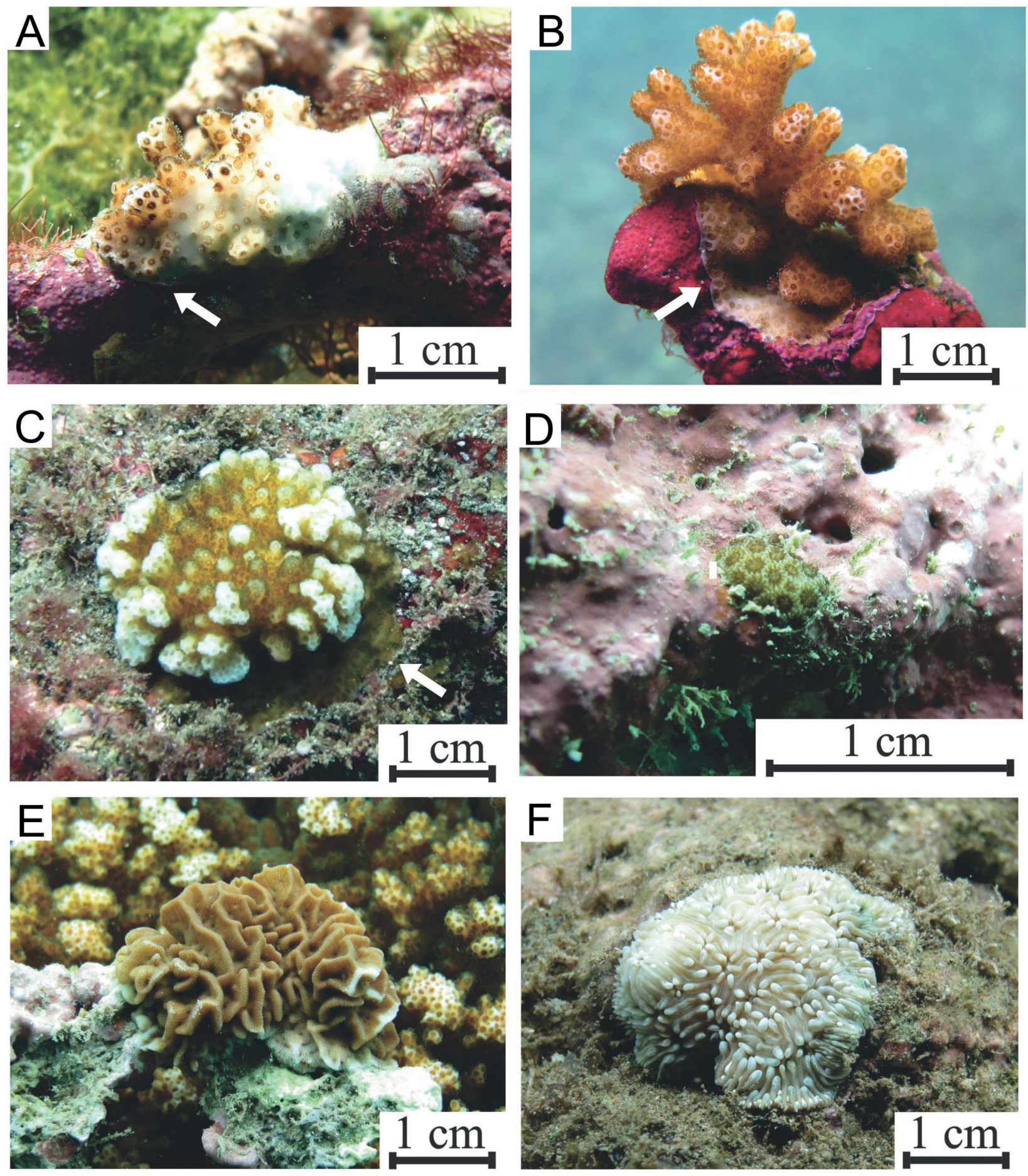

Figura 2. Corales juveniles producto de reproducción sexual asentados sobre sustratos naturales en Isla Gorgona (Pacífico Tropical Oriental). Pocillopora sp. asentado sobre escombros de coral con algas calcáreas (A, B); Pocillopora sp. asentado sobre una pared rocosa con algas filamentosas (C); Porites $\mathrm{cf}$. panamensis asentado sobre escombros de coral con algas calcáreas (D); Pavona varians asentado sobre escombros de coral con algas calcáreas (E); Pavona gigantea asentado sobre una pared rocosa con algas filamentosas (F). Las flechas blancas señalan la base incrustante en los ejemplares de pocilopóridos.
Figure 2. Coral juveniles derived from sexual reproduction attached to natural substrates at Gorgona Island (Eastern Tropical Pacific). Pocillopora sp. attached to coral rubble with calcareous algae (A, B); Pocillopora sp. attached to rocky wall with filamentous algae (C); Porites cf. panamensis attached to coral rubble with calcareous-algae (D); Pavona varians attached to coral rubble with calcareous-algae (E); Pavona gigantea attached to coral rubble with calcareous-algae (E); Pavona gigantea attached to rocky wall with filamentous algae (F). White arrows point to encrusting base in pocilloporid specimens. 


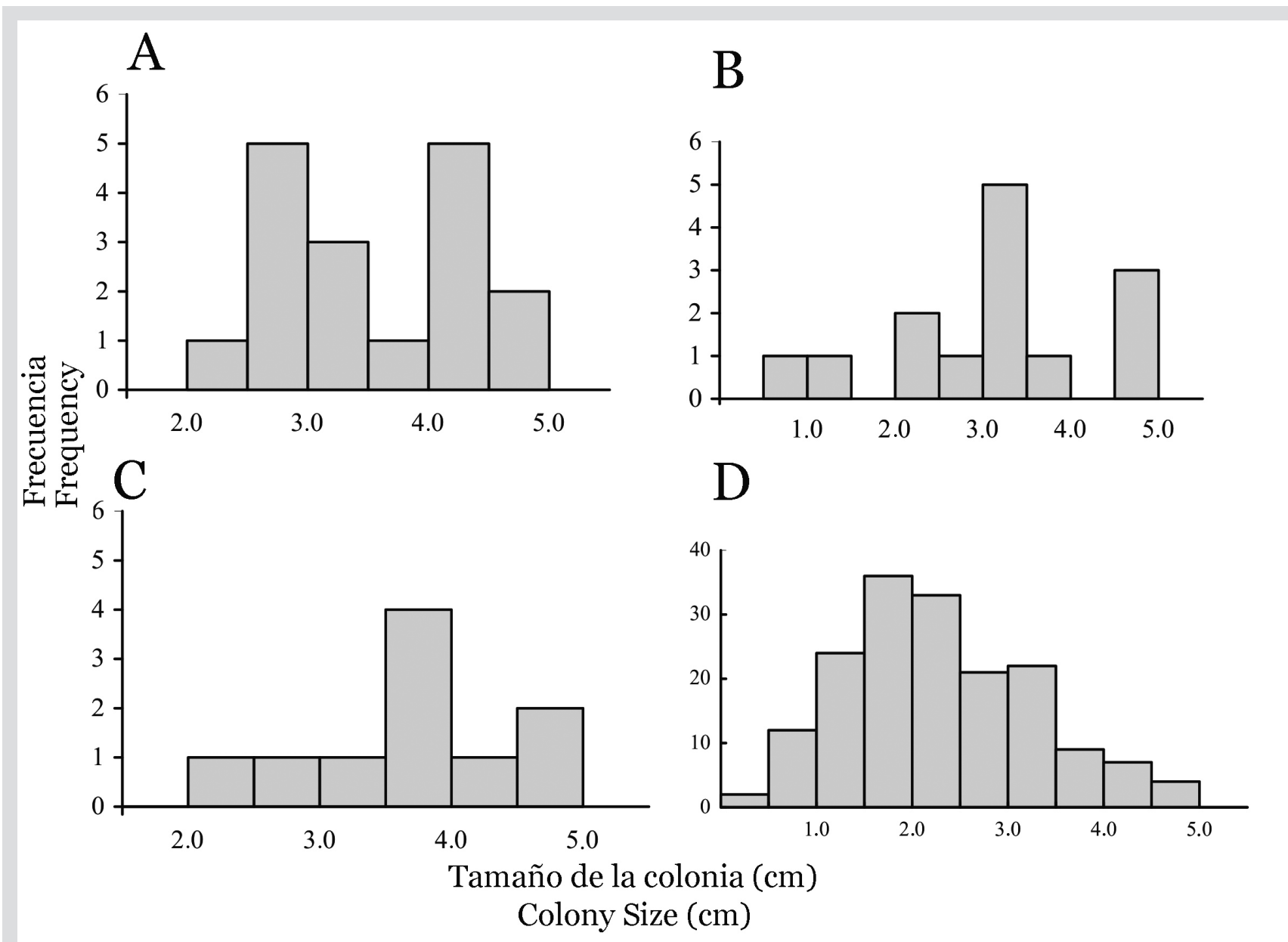

Figura 3. Distribuciones de frecuencia del tamaño de los corales juveniles (diámetro más largo de una colonia vista desde arriba) en Isla Gorgona (Pacífico Tropical Oriental). Las distribuciones se truncan a $5,0 \mathrm{~cm}$, ya que los corales juveniles fueron definidos como colonias $\leq 5,0 \mathrm{~cm}$. Distribución para los corales juveniles pocilopóridos $(\mathrm{N}=41)$ en tres sitios: La Azufrada (A), El Laberinto (B) y Playa Blanca (C). Distribución para corales juveniles de Porites panamensis $(\mathrm{N}=170)$ en El Arrecifito (D).
Figure 3. Size frequency distributions of coral juveniles (largest diameter of a colony observed from the top) at Gorgona Island (Eastern Tropical Pacific). The distributions are truncated at $5.0 \mathrm{~cm}$, since juveniles are defined as colonies $\leq 5.0 \mathrm{~cm}$. Distribution for pocilloporid juveniles $(\mathrm{N}=41)$ at three sites: La Azufrada (A), El Laberinto (B), and Playa Blanca (C). Distribution for Porites panamensis juveniles $(\mathrm{N}=170)$ at El Arrecifito (D).

\section{DISCUSIÓN}

Este estudio registra por primera vez en el Pacífico colombiano, evidencia del reclutamiento de corales provenientes de reproducción sexual. Sin embargo, se obtuvieron dos resultados contradictorios similares a los descritos en estudios previos sobre el reclutamiento de corales en el PTO: a pesar de las bajas densidades, se encontraron corales juveniles en los sustratos naturales de arrecifes de coral y sitios rocosos, pero el método de muestreo habitual de las placas de asentamiento no detectó reclutamiento coralino (Birkeland, 1977; Wellington, 1982; Richmond, 1985; Medina-Rosas et al., 2005; López-Pérez et al., 2007; LozanoCortés y Zapata, 2014). En los arrecifes de coral, los corales juveniles se encontraron asentados sobre los escombros de
In addition to other reef locations in the ETP where juveniles of Pocillopora sp. and Porites panamensis derived from sexual reproduction have been already observed on natural substrates (Richmond, 1985; MedinaRosas et al., 2005; Glynn et al., 1991; 1994; 2017a), this study reports the abundance and size distribution of coral juveniles for these species at the Colombian Pacific. The abundance of coral recruitment on natural substrates in the ETP reefs has been very low, indicating that the abundance reported here for $P$. panamensis is one of the highest in the region, second to that of Pavona clavus in Costa Rica (Glynn et al., 2017a), and the result of a recruitment pulse and a brooding reproductive mode (Glynn et al., 1994). 
coral o en la matriz coralina consolidada cubierta por algas calcáreas, mientras que, en los sitios rocosos, los corales juveniles se encontraron asentados sobre paredes rocosas, desnudas o parcialmente cubiertas por algas filamentosas.

Además de constituir nuevos sitios del PTO en los que han sido observadas colonias juveniles de Pocillopora sp. y Porites panamensis producto de la reproducción sexual sobre sustratos naturales (Richmond, 1985; Medina-Rosas et al., 2005; Glynn et al., 1991, 1994, 2017a), adicionalmente, este estudio describe la distribución de la abundancia y del tamaño de los juveniles para estas especies en el Pacífico colombiano. Los valores del reclutamiento de corales sobre sustratos naturales en los arrecifes del PTO han sido muy bajos, indicando que la abundancia aquí registrada de juveniles de $P$. panamensis es una de las cifras más altas conocidas hasta el momento en la región, siendo superado solo por la abundancia de juveniles de Pavona clavus en Costa Rica (Glynn et al., 2017a) y seguramente resultado de un pulso de reclutamiento de un modo reproductivo incubador (Glynn et al., 1994).

La implicación de los resultados del reclutamiento de corales obtenidos hasta ahora en el PTO es doble. Primero, los corales sí pueden reproducirse sexualmente en la región, lo cual ya había sido previamente discutido por varios estudios histológicos sobre la reproducción del coral a lo largo del PTO (Glynn et al., 2017a y referencias incluidas). Estos resultados rechazan la hipótesis de que los corales sean estériles en la región (Richmond, 1985, 1987; Glynn et al., 1991), y al mismo tiempo rebaten la idea de que el reclutamiento derive principalmente de la fragmentación de las colonias (Birkeland, 1977; Highsmith, 1982; Wellington, 1982; Richmond, 1985).

En segundo lugar, la escasez del reclutamiento de corales en sustratos artificiales en el PTO puede ser debido a algún factor desconocido que impide que las larvas de coral se asienten o sobrevivan sobre los sustratos artificiales muestreados. Los resultados negativos obtenidos con las placas de asentamiento podrían estar relacionados con: 1) el uso de materiales de sustrato inapropiados, 2) tiempo insuficiente después de la instalación para un adecuado acondicionamiento del sustrato (López-Pérez et al., 2007), y 3) competencia, depredación o herbivoría en las placas de asentamiento (Birkeland, 1977; Sammarco, 1982, 1985; Richmond, 1987). Sin embargo, en este estudio, la primera causa es una explicación poco probable porque se utilizaron cinco materiales diferentes, incluyendo la cerámica, la cual ha producido los mejores resultados de reclutamiento tanto en el Indo-Pacífico (Harriott y Fisk, 1987) como en el Caribe (Tomascik, 1991). En el segundo caso, es bien sabido que los sustratos artificiales requieren un período de acondicionamiento durante el cual se desarrolla una comunidad incrustante antes de que ocurra el asentamiento de
The implication of coral recruitment results obtained so far in the ETP, is twofold: first, corals are able of reproducing sexually in the region, previously suggested by several histological studies about coral reproduction throughout the ETP (Glynn et al., 2017a and references therein). These results refute the hypothesis that corals in the ETP are sterile (Richmond, 1985; 1987; Glynn et al., 1991), and at the same time challenge the idea that recruitment derives mostly from fragmentation of colonies (Birkeland, 1977; Highsmith, 1982; Wellington, 1982; Richmond, 1985).

Second, the scarcity of coral recruitment on artificial substrates in the ETP must then be due to some unknown factor that prevents coral larvae from settling or surviving on artificial sampling substrates. The negative results obtained with the settlement plates could be related to: 1) the use of inappropriate substrate materials, 2) insufficient time after deployment for adequate substrate conditioning (López-Pérez et al., 2007), and 3) competition, predation, or herbivory, on settlement plates (Birkeland, 1977; Sammarco, 1982; 1985; Richmond, 1987). However, in this study the first is an unlikely explanation because we used five different materials, including ceramic that has yielded the highest recruitment results in other studies, both in the Indo-Pacific (Harriott and Fisk, 1987) and the Caribbean (Tomascik, 1991). In the second case, it is well known that artificial substrates require a conditioning period during which an encrusting community develops before coral larvae will settle (Segal et al., 2012). While the duration of the conditioning period is not well established and may be highly variable from place to place, in southwestern Mexico coral recruitment occurred 5-10 months after plates had been submerged (López-Pérez et al., 2007). Since our plates were submerged for six months, it is plausible that the encrusting community that induces or enhances coral settlement had not fully developed. However, in a previous attempt to document recruitment on artificial substrates at La Azufrada reef, the settlement plates were submerged for a full year and yet no recruits were found (Lozano-Cortés and Zapata, 2014). In the third case, it is known that competition, predation, or herbivory on settlement plates may prevent coral spats from surviving and actually recruiting on these substrates (Birkeland, 1977; Sammarco, 1982; 1985; Díaz-Pulido et al., 2010). 
las larvas de coral (Segal et al., 2012). Aunque la duración del período de acondicionamiento no está bien establecida y puede variar mucho de un lugar a otro, en el sudoeste de México el reclutamiento de corales tuvo lugar de 5 a 10 meses después de sumergir las placas (López-Pérez et al., 2007). Dado que nuestras placas se mantuvieron sumergidas durante poco más de seis meses, es razonable pensar que la comunidad incrustante que induce o mejora el asentamiento de los corales aún no se hubiera desarrollado completamente. Sin embargo, en un intento previo de documentar el reclutamiento en placas de asentamiento en el mismo arrecife (La Azufrada), las placas fueron sumergidas durante un año completo y aun así no se encontraron reclutas (Lozano-Cortés y Zapata, 2014). En el tercer caso, se sabe que la competencia, la depredación, o la herbivoría en las placas de asentamiento, pueden evitar que los reclutas de coral sobrevivan y se produzca un verdadero reclutamiento sobre estos sustratos (Birkeland, 1977; Sammarco, 1982, 1985; Díaz-Pulido et al., 2010). Aunque se eliminaron periódicamente algas y otros invertebrados sésiles de la mitad de las placas para reducir la posible competencia con los potenciales reclutas coralinos, con frecuencia se observaron erizos de mar, ophiuroides, y gasterópodos sobre las placas, que pudieron haber interferido con el asentamiento o supervivencia del coral.

Además, existe un argumento metodológico razonable relacionado con el tamaño de la muestra, cuya solución puede ser imposible en la práctica: si la abundancia del reclutamiento de corales es naturalmente baja, entonces el área o el esfuerzo de muestreo debería ser considerablemente mayor que la utilizada para poder detectar con éxito los pocos reclutas de coral. Si bien existen otras diversas posibles explicaciones, ninguna de ellas es lo suficientemente general como para explicar el fracaso común para documentar el reclutamiento de corales en placas de asentamiento en el PTO, y, por lo tanto, la razón para estos resultados sigue sin estar clara.

En conclusión, los resultados presentados en este estudio indican que existe reclutamiento de corales producto de la reproducción sexual en Isla Gorgona. Esto, sin embargo, ha sido pasado por alto por la falta de observaciones de asentamiento coralino en sustratos artificiales, y la dificultad para diferenciar a los corales juveniles producto de la reproducción sexual de aquellos que son producto de la sobrevivencia de fragmentos de coral. Las futuras investigaciones requieren examinar la contribución relativa de la reproducción sexual y asexual al reclutamiento coralino, y su papel en la recuperación y el mantenimiento de las poblaciones coralinas y los arrecifes en el Pacífico colombiano y el PTO.
Although we periodically removed algae and other sessile invertebrates from half of the plates to reduce potential competition with recently settled corals, organisms like sea urchins, ophiuroids, and gastropods were commonly seen on the plates and may have interfered with coral settlement or survival.

Additionally, there is a reasonable methodological argument related to the sampling size, whose solution can be impossible in practice: if the values of coral recruitment are naturally low, then the sampling area should be considerably larger than the one we used to successfully detect coral recruits. While there are several other potential explanations, none of them are general enough to explain the common failure to document coral recruitment on artificial settlement plates in the ETP, and therefore the reason for these results remains obscure.

In conclusion, the results presented here indicate that coral recruitment derived from sexual reproduction occurs at Gorgona Island. This, however, has been overlooked by the failure to observe coral settlement on artificial sampling substrates, and the difficulty to differentiate sexual juveniles from small surviving coral fragments. Future research requires examination of the relative contribution of sexual and asexual reproduction to the supply of coral recruitment and their role in the recovery and maintenance of coral populations and coral reefs in the Colombian Pacific and ETP.

\section{ACKNOWLEDGEMENTS}

We are thankful to National Natural Parks of Colombia for the constant support to our studies, this research would have been impossible without the help of the staff of Henry von Prahl scientific research station at PNN Gorgona. Main funding provided by COLCIENCIAS and Universidad del Valle (Project code: 1106-489-25135); CGM was beneficiary of the COLCIENCIAS doctorate program. 


\section{AGRADECIMIENTOS}

Se agradece a Parques Nacionales Naturales de Colombia por su apoyo constante a nuestros estudios; esta investigación hubiera sido imposible sin la ayuda del personal de la estación de investigación científica Henry von Prahl en el PNN Gorgona. Proyecto co-financiado por COLCIENCIAS y Universidad del Valle (Código de proyecto: 1106-48925135); CGM fue beneficiario del programa para doctorados de COLCIENCIAS.

\section{BIBLIOGRAFÍA / LITERATURE CITED}

Babcock, R., A. Baird, S. Piromvaragorn, D. Thomson and B. Willis. 2003. Identification of scleractinian coral recruits from Indo-Pacific reefs. Zool. Stud., 42: 211-226.

Birkeland, C. 1977. The importance of rate of biomass accumulation in early successional stages of benthic communities to the survival of coral recruits. Proceedings of the Third International Coral Reef Symposium, 1: 15-21.

Blanco, J.F. 2009. The hydroclimatology of Gorgona Island: Seasonal and ENSO-related patterns. Actual. Biol., 31: 111-121.

Cabral-Tena, R.A., H. Reyes-Bonilla, S. Lluch-Cota, D. Paz-García, L. Calderón-Aguilera, O. Norzagaray-Lopez and E. Balari. 2013. Different calcification rates in males and females of coral Porites panamensis in the Gulf of California. Mar. Ecol. Prog. Ser., 476: 1-8.

Caley, M., M. Carr, M. Hixon, T. Hughes, G. Jones and B. Menge. 1996. Recruitment and the local dynamics of open marine populations. An. Rev. Ecol. Syst., 27: 477-500

Castrillón, A., C. Muñoz and F. Zapata. 2015. Reproductive patterns of the coral Pocillopora damicornis at Gorgona Island, Colombian Pacific Ocean. Mar. Biol. Res., 11: 1065-1075.

Castrillón-Cifuentes, A.L., D.F. Lozano-Cortés and F.A. Zapata. 2017. Effect of short-term subaerial exposure on the cauliflower coral, Pocillopora damicornis, during a simulated extreme low-tide event. Coral Reefs, 36(2): 401-414.

Díaz, J., J. Pinzón, A. Perdomo, L. Barrios y M. López-Victoria. 2001. Generalidades. 17-26. En: Barrios, L. y M. López-Victoria (Eds.). Gorgona marina, contribución al conocimiento de una isla única. INVEMAR, Serie de Publicaciones Especiales No. 7. Santa Marta, Colombia. 160 p.

Diaz-Pulido, G., S. Harii, L. McCook and O. Hoegh-Guldberg. 2010. The impact of benthic algae on the settlement of a reef-building coral. Coral Reefs, 29: 203-208.

Edmunds, P. J. 2004. Juvenile coral population dynamics track rising seawater temperature on a Caribbean reef. Mar. Ecol. Prog. Ser., 269: 111-119.

Fiedler, P. and M. Lavin. 2017. Oceanographic conditions of the Eastern Tropical Pacific. 59-83. In: Glynn P. W., D.P. Manzello and I.C. Enochs (Eds.).Coral Reefs of the Eastern Tropical Pacific: Persistence and Loss in a Dynamic Environment (Coral Reefs of the World, Volume 8). Springer, Dordrecht. $675 \mathrm{p}$.

Giraldo, A., E. Rodríguez-Rubio y F. Zapata. 2008. Condiciones oceanográficas de Isla Gorgona, Pacifico Oriental Tropical de Colombia. Lat. Am. J. Aquat. Res., 36: 121-128.

Glynn, P. W., J. S. Ault, G. M. Wellington, N. J. Gassman, C. M. Eakin, J. Cortés, D. B. Smith and H. M. Guzmán. 1991. Reef coral reproduction in the eastern Pacific: Costa Rica, Panama and Galápagos Islands (Ecuador). Part I. Pocilloporidae. Mar. Biol., 109: 355-368.

Glynn, P. W., S. B. Colley, C. M. Eakin, D. B. Smith, J. Cortés, N. J. Gassman and J.S. Feingold. 1994. Reef coral reproduction in the eastern Pacific: Costa Rica, Panamá, and Galápagos Islands (Ecuador). II. Poritidae. Mar. Biol., 118(2): 191-208.

Glynn, P., S. Colley, N. Gassman, K. Black, J. Cortés and J. Maté. 1996. Reef coral reproduction in the eastern Pacific: Costa Rica, Panama, and Galapagos Islands (Ecuador). III. Agariciidae (Pavona gigantea and Gardineroseris planulata). Mar. Biol., 125: 579-601.

Glynn, P., S. Colley, E. Carpizo-Ituarte and R. Richmond. 2017a. Coral reproduction in the Eastern Pacific. In: Glynn, P., D. Manzello and I. Enochs (Eds.). Coral Reefs of the Eastern Tropical Pacific: Persistence and loss in a dynamic environment (Coral Reefs of the world, Volume 8). Springer, Dordrecht, Germany. 657 p.

Glynn, P. W., J.J Alvarado, S. Banks, J. Cortés, J.S. Feingold, C. Jiménez... and S. Navarrete. 2017b. Eastern Pacific coral reef provinces, coral community structure and composition: an overview: 107-176. In: Glynn, P., D. Manzello and I. Enochs (Eds.). Coral Reefs of the Eastern Tropical Pacific: Persistence and loss in a dynamic environment (Coral Reefs of the world, Volume 8). Springer, Dordrecht, Germany. 657 p.

Harriott, V. and D. Fisk. 1987. A comparison of settlement plate types for experiments on the recruitment of scleractinian corals. Mar. Ecol. Prog. Ser., 37: 201-208. 
Highsmith, R. 1982. Reproduction by fragmentation in corals. Mar. Ecol. Prog. Ser., 7: 207-226.

Hughes, T. and J. Tanner. 2000. Recruitment failure, life histories, and long-term decline of Caribbean corals. Ecology, 81: $2250-2263$.

Hughes, T., N. Graham, J. Jackson, P. Mumby and R. Steneck. 2010. Rising to the challenge of sustaining coral reef resilience. Trends Ecol. Evol., 25: 633-642.

López-Pérez, R., M. Mora-Pérez and G. Leyte-Morales. 2007. Coral (Anthozoa: Scleractinia) recruitment at Bahías de Huatulco, Western México: Implications for coral community structure and dynamics. Pac. Sci., 61: 355-369.

Lozano-Cortés, D. and F. Zapata. 2014. Invertebrate colonization on artificial substrates in a coral reef at Gorgona Island, Colombian Pacific Ocean. Rev. Biol. Trop., 62 (Suplem. 1): 161-168.

Medina-Rosas, P., J. Carriquiry y A. Cupul-Magaña. 2005. Reclutamiento de Porites (Scleractinia) sobre sustrato artificial en arrecifes afectados por El Niño 1997-98, en Bahía de Banderas, Pacífico mexicano. Cienc. Mar., 31: 103-109.

Muñoz, C. G. y F. A. Zapata. 2013. Plan de manejo de los arrecifes coralinos del Parque Nacional Natural Gorgona. Parques Nacionales Naturales y WWFColombia. Cali, Colombia. 68 p.

Muñoz, C. G., C. Wild and F. A. Zapata. 2015. Drift logs are effective agents of physical coral fragmentation in a tropical eastern Pacific coral reef. B. Mar. Sci., 91(3): 375-376.

Palacios, M. M., C.G. Muñoz and F.A. Zapata. 2014. Fish corallivory on a pocilloporid reef and experimental coral responses to predation. Coral Reefs, 33 : 625-636.

Richmond, R. 1985. Variations in the population biology of Pocillopora damicornis across the Pacific. Proceedings of the 5th International Coral Reef Congress, Tahiti, 6: 101-106.

Richmond, R.H. 1987. Energetic relationships and biogeographical differences among fecundity, growth and reproduction in the reefs: coral Pocillopora damicornis. Bull. Mar. Sci., 41(2): 594-604.

Richmond, R. 1997. Reproduction and recruitment in corals: Critical links in the persistence of reefs. 175-197. In: Birkeland, C. (Ed.). Life and Death of Coral Reefs. Chapman and Hall, New York. 536 p.

Sammarco, P.W. 1982. Effects of grazing by Diadema antillarum Philippi (Echinodermata: Echinoidea) on algal diversity and community structure. Journal of Exp. Mar. Biol. and Ecol., 65(1): 83-105.

Sammarco, P. 1985. The Great Barrier Reef vs. the Caribbean: Comparisons of grazers, coral recruitment patterns and reef recovery. Proceedings of the 5th International Coral Reef Congress, Tahiti. 4: 391-397.

Schmidt-Roach, S., K. J. Miller, P. Lundgren, and N. Andreakis. 2014. With eyes wide open: a revision of species within and closely related to the Pocillopora damicornis species complex (Scleractinia; Pocilloporidae) using morphology and genetics. Zool. J. Linnean Soc., 170(1): 1-33.

Segal, B., V. Berenguer and C. Castro. 2012. Experimental recruitment of the Brazilian endemic coral Mussismilia braziliensis and conditioning of settlement plates. Cienc. Mar., 38(1A): 1-10.

Tomascik, T. 1991. Settlement patterns of Caribbean scleractinian corals on artificial substrata along a eutrophication gradient, Barbados, West Indies. Mar. Ecol. Prog. Ser., 77: 261-269.

Wellington, G. 1982. Depth zonation of corals in the Gulf of Panama: Control and facilitation by resident reef fishes. Ecol. Monogr., 52: $224-241$.

Williams, D.E., M.W. Miller and K.L. Kramer. 2008. Recruitment failure in Florida Keys Acropora palmata, a threatened Caribbean coral. Coral Reefs, 27(3): 697-705.

Zapata, F. 2017. Temporal dynamics of coral and algal cover and their drivers on a coral reef of Gorgona Island, Colombia (Eastern Tropical Pacific). Rev. Acad. Col. Cienc. Ex. Fís. Nat., 41(160): 298-310.

Zapata, F. and B. Vargas-Ángel. 2003. Corals and coral reefs of the Pacific coast of Colombia. 419-447. In: Cortés, J. (Ed.). Latin American Coral Reefs. Elsevier Science B.V., Amsterdam. 508 p. 